\title{
Artroplastia do ombro no tratamento das fraturas da extremidade proximal do úmero: Conceitos atuais*
}

\section{Shoulder arthroplasty for the treatment of proximal humeral fractures: current concepts}

\author{
Geraldo da Rocha Motta Filho ${ }^{10}$ Marcus Vinícius Galvão Amaral ${ }^{1}$ \\ ${ }^{1}$ Centro de Cirurgia do Ombro e Cotovelo, Instituto Nacional de \\ Traumatologia e Ortopedia (INTO), Rio de Janeiro, RJ, Brasil \\ Endereço para correspondência Geraldo da Rocha Motta Filho, MD, \\ MSc, Rua Raimundo Magalhães, 92, Gávea, Rio de Janeiro, \\ RJ, 22451-150, Brasil (e-mail: geraldomotta@terra.com.br).
} Rev Bras Ortop 2022;57(4):529-539.

\section{Resumo \\ Palavras-chave \\ - artroplastia \\ - ombro \\ - fraturas ósseas}

Fraturas complexas da extremidade proximal do úmero, especialmente em idosos, frequentemente necessitam de tratamento cirúrgico artroplástico. Tradicionalmente, a hemiartroplastia (HA) do ombro é o método de escolha, com longa sobrevida do implante, e oferece um ombro indolor, mas com resultados clínicos heterogêneos, relacionados ao correto posicionamento do implante quanto à altura e à versão, além da consolidação anatômica dos tubérculos. Atualmente, a utilização de artroplastias reversas do ombro para o tratamento dessas fraturas vêm aumentando exponencialmente, com melhores resultados funcionais do que as HAs, principalmente quanto à flexão anterior, apesar de a longevidade do implante ainda não ter sido estabelecida. O desenvolvimento de componentes umerais protéticos específicos para o tratamento de fraturas, introduzidos na prática clínica nos últimos anos, levou a resultados clínicos melhores.

Complex proximal humeral fractures, especially in elderly patients, often require arthroplastic surgical treatment. Traditionally, shoulder hemiarthroplasty (HA) is the method of choice, resulting in long implant survival and a painless shoulder; however, shoulder HA has heterogeneous clinical outcomes related to the correct position of the implant, both in terms of height and version, and the anatomical consolidation of tuberosities. Today, reverse shoulder arthroplasties are increasingly used to treat such fractures. These techniques result in better functional outcomes compared to HAs, especially regarding anterior flexion, but implant longevity has not been established. The development of specific prosthetic humeral components for the treatment of fractures, which were recently introduced in the clinical practice, led to better clinical outcomes.
Trabalho desenvolvido no Centro de Cirurgia do Ombro e Cotovelo, Instituto Nacional de Traumatologia e Ortopedia (INTO), Rio de Janeiro, RJ, Brasil. recebido

24 de Agosto de 2019

aceito

16 de Setembro de 2020

Publicado on-line

Março 31, 2021
DOI https://doi.org/

$10.1055 / \mathrm{s}-0040-1721359$.

ISSN 0102-3616. (c) 2021. Sociedade Brasileira de Ortopedia e Traumatologia. All rights reserved.

This is an open access article published by Thieme under the terms of the Creative Commons Attribution-NonDerivative-NonCommercial-License, permitting copying and reproduction so long as the original work is given appropriate credit. Contents may not be used for commercial purposes, or adapted, remixed, transformed or built upon. (https://creativecommons.org/ licenses/by-nc-nd/4.0/)

Thieme Revinter Publicações Ltda., Rua do Matoso 170, Rio de Janeiro, RJ, CEP 20270-135, Brazil 


\section{Introdução}

Fraturas da extremidade proximal do úmero representam até $10 \%$ de todas as fraturas que acometem a população idosa. ${ }^{1}$ Muitas dessas fraturas podem ser tratadas de forma incruenta com resultados satisfatórios. Por outro lado, fraturas complexas, como aquelas em três e quatro partes, de acordo com a classificação de Neer, ${ }^{2}$ fraturas-luxações, fraturas articulares da cabeça umeral, e fraturas com desvio associadas a múltiplos fragmentos e perda óssea metafisária, frequentemente necessitam de tratamento cirúrgico (-Fig. 1A-B). ${ }^{2}$

Tradicionalmente, a hemiartroplastia (HA) é indicada para as fraturas em que é impossível se obter uma redução anatômica, e quando é evidenciado um alto risco de falha da fixação interna. ${ }^{1,3,4}$

A HA é tecnicamente difícil, principalmente no que se refere à restituição do comprimento do úmero e à versão apropriada da cabeça umeral. ${ }^{5} \mathrm{Um}$ dos fatores que determinam a qualidade do resultado clínico é a reconstrução da anatomia do úmero proximal com o reparo e consolidação dos tubérculos maior e menor. ${ }^{6,7}$

O desenvolvimento de novas técnicas de fixação dos tubérculos e de componentes umerais específicos para o tratamento artroplástico propiciaram uma melhoria dos resultados clínicos. Esses componentes umerais com baixo perfil, ou seja, menor espessura metálica proximal, com offset medializado e presença de orifícios para passagem de suturas no colo da haste, favorecem o posicionamento anatômico do tubérculo maior, a colocação de enxerto ósseo, e a sutura dos mesmos. ${ }^{8}$ (-Fig. 2).

Ao longo dos anos, os resultados das HAs não se revelaram consistentes na prática clínica. ${ }^{7-10}$ A introdução das artroplastias reversas do ombro (AROs) no tratamento de doenças degenerativas do ombro, com resultados entusiasmantes,

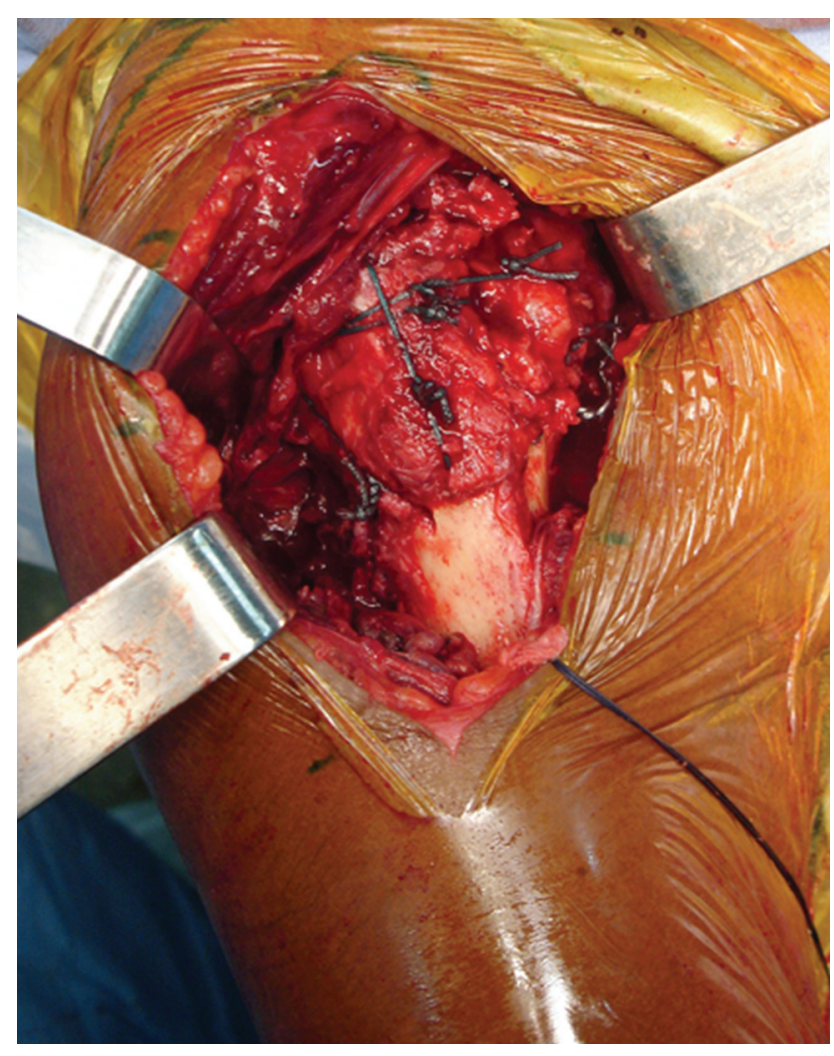

Fig. 2 Imagem perioperatória do amarrilho dos tubérculos maior e menor associados à hemiartroplastia.

estimulou a comunidade de especialistas a ampliar o seu uso para o tratamento de fraturas complexas da extremidade proximal do úmero. ${ }^{11,12}$

A avaliação dos resultados de 69.120 fraturas, tratadas entre os anos de 2008 e 2016 no Korean Health Insurance
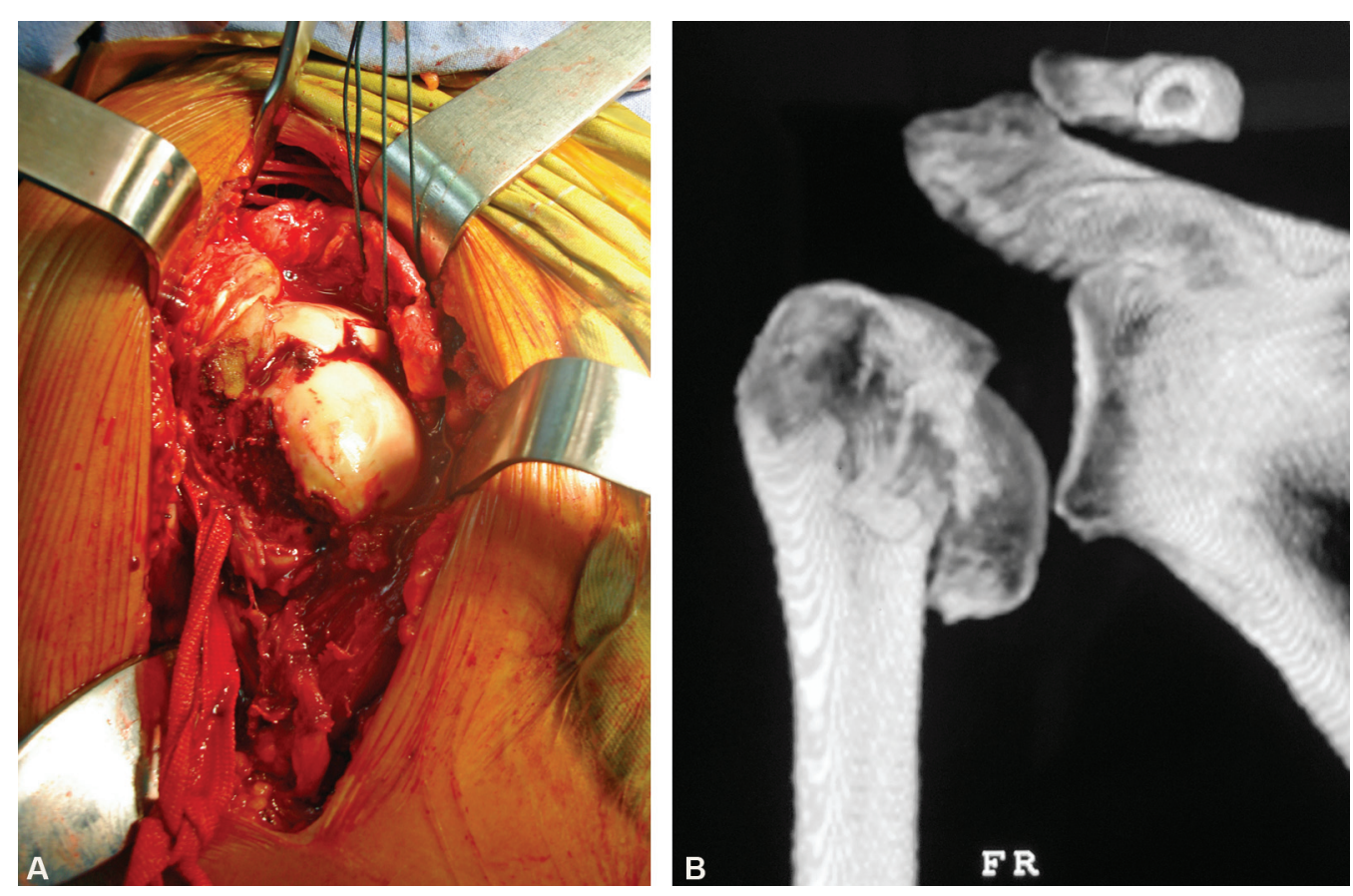

Fig. 1 (A) Imagem perioperatória de um head split. (B) Imagem de tomografia computadorizada em três dimensões (3D) de um head split. 
Review and Assessment Service, ${ }^{13}$ demonstrou aumento significativo da realização de tratamento cirúrgico das fraturas da extremidade proximal do úmero, que subiu de $24,6 \%$ em 2008 para $36,8 \%$ em 2016 . No total, $85,6 \%$ dessas fraturas foram tratadas com redução cirúrgica e fixação interna. Nessa casuística, a indicação de artroplastia no tratamento dessas fraturas variou discretamente, de 8,6\% em 2008 para $9,9 \%$ em 2016. Porém, apesar da pequena variação no total de artroplastias realizadas nesse grupo de pacientes, a incidência do uso da ARO aumentou significativamente, de $8,2 \%$ em 2008 para 52\% em 2016, principalmente nos idosos acima dos 80 anos de idade, demonstrando uma substituição no uso das técnicas de artroplastia para o tratamento dessas fraturas. $^{13}$

O objetivo deste trabalho é rever o que existe de atual na literatura acerca da utilização das HAs e AROs no tratamento das fraturas desviadas da extremidade proximal do úmero, ou seja, em que não são factíveis a redução cirúrgica e a fixação interna.

\section{Critérios para indicação e contraindicação da hemiartroplastia e da artroplastia reversa}

Desde os relatos iniciais de Neer, na década de $1970,{ }^{14}$ a substituição protética da extremidade proximal do úmero para o tratamento das fraturas complexas dessa articulação foi bem aceita. ${ }^{7-10}$ Porém, ao longo dos anos e com a introdução de escores de avaliação clínica mais específicos, os resultados funcionais se revelaram muitas vezes imprevisíveis, inconsistentes, e inferiores aos obtidos em pacientes com doenças degenerativas e inflamatórias submetidos a artroplastia total do ombro (ATO). ${ }^{15}$

A HA da extremidade proximal do úmero é indicada classicamente em indivíduos idosos com baixa ou moderada demanda funcional, ou ainda em pacientes em torno dos 60 anos em que não seja possível realizar redução aberta e fixação interna (RAFI) da fratura, e que tenham preferencialmente o tubérculo maior pouco fragmentado. ${ }^{7-10}$ Nos dias de hoje, podem ser utilizados componentes umerais específicos para o tratamento de fraturas que irão favorecer a consolidação dos tubérculos, e que permitam a conversão futura para uma ARO sem que seja necessária a sua substituição. ${ }^{7,8,16}$ Apesar dos resultados desfavoráveis das artroplastias constritas no passado, nos anos 1980, Paul Grammont redesenhou esse modelo de prótese do ombro, com modificações biomecânicas que melhoraram os resultados clínicos e reduziram o número de complicações. Dessa forma, esse modelo de prótese se popularizou. $^{17,18}$

A utilização das AROs no tratamento das fraturas tem, nos dias de hoje, suplantado as HAs em virtude da expectativa de que os resultados clínicos sejam mais consistentes. Pacientes com idade superior a 70 anos que apresentem fraturas desviadas em 3 e 4 partes constituem esse potencial grupo de $^{\text {pacientes. }}{ }^{12}$

São condições necessárias à realização da ARO a estrutura e função preservadas do músculo deltoide. Porém, o deltoide hipotrofiado não seria um impedimento à realização de uma ARO, desde que a sua inervação esteja preservada. ${ }^{18}$ Já a presença da paralisia completa do nervo axilar é contraindi- cação clássica pelo alto risco de instabilidade e potencial limitado de melhora funcional. ${ }^{17,18}$ Estrutura e estoque ósseo adequados na glenoide, que permitem a fixação segura do componente, são condições essenciais para a realização do procedimento. Nas situações em que exista erosão ou perda da massa óssea, a decisão deverá ser baseada em imagens de tomografia computadorizada tridimensional. São contraindicações absolutas: infecção ativa, neuroartropatia, e grave perda óssea na glenoide. Pacientes com osteopenia intensa, como usuários crônicos de corticoesteróides, têm contraindicação relativa. ${ }^{17,18}$

Apesar do uso da HA no tratamento das fraturas, o sucesso de seus resultados é questionável. ${ }^{7-10}$ Frequentemente, o resultado das HAs no tratamento de fraturas é pouco previsível, com alto índice de complicações relacionadas aos tubérculos. $^{7-10}$ Estudos recentes têm demonstrado melhores resultados no uso da ARO do que da HA no tratamento de fraturas desviadas da extremidade proximal do úmero na população idosa. ${ }^{19-30}$ Essa diferença ocorre porque o resultado funcional da HA no tratamento de fraturas é diretamente relacionado à consolidação anatômica dos tubérculos. ${ }^{7-10}$ Já na no tratamento das fraturas com ARO, ocorrem resultados funcionais mais homogêneos, mesmo em situações em que não ocorre consolidação, ou ocorre consolidação viciosa dos tubérculos. $^{11,12,31-35}$

Em situações clínicas de fraturas com tempo de evolução superior a três semanas e sequelas de fraturas do úmero, nas quais as HAs apresentam péssimos resultados em virtude da necessidade de dissecção e mobilização mais agressiva dos tubérculos, o que influencia negativamente sua consolidação, as AROs, ainda sim, podem oferecer bons resultados. ${ }^{21,36,37}$ Dessa forma, são uma alternativa para pacientes idosos que apresentam resultados insatisfatórios após o tratamento não operatório inicial. ${ }^{36,37}$

\section{Avaliação dos resultados e análise da literatura}

Os resultados funcionais associados às HAs do ombro para fraturas são inconsistentes. ${ }^{7-10}$ Isso se deve aos complexos fatores técnicos relacionados à reconstrução, ao momento da cirurgia, às características da população, além da heterogenicidade dos escores clínicos de avaliação. ${ }^{7-10}$ Apesar disso, os resultados revelam uma taxa de alívio da dor de $73 \%$ a $97 \%$, e um percentual de satisfação subjetiva dos pacientes de $70 \%$ a $92 \%$ dos casos. ${ }^{7-10}$

Um posicionamento preciso da HA em uma fratura complexa da extremidade proximal do úmero, com reprodução da altura e versão do úmero, permitirá uma redução anatômica e fixação rígida dos tubérculos, o que é essencial para um resultado clínico satisfatório. .,7,8 $^{2}$

As séries de $\operatorname{casos}^{7,10,15}$ publicadas que avaliam esse procedimento demonstram que os resultados funcionais correlacionam-se diretamente com a consolidação anatômica dos tubérculos.

No plano vertical, uma medida com valor prognóstico para avaliação do correto posicionamento do tubérculo maior é a distância cabeça-tubérculo (DCT), que é uma medida objetiva da altura do tubérculo maior em relação à superfície articular da cabeça umeral da prótese. Usualmente, essa distância 

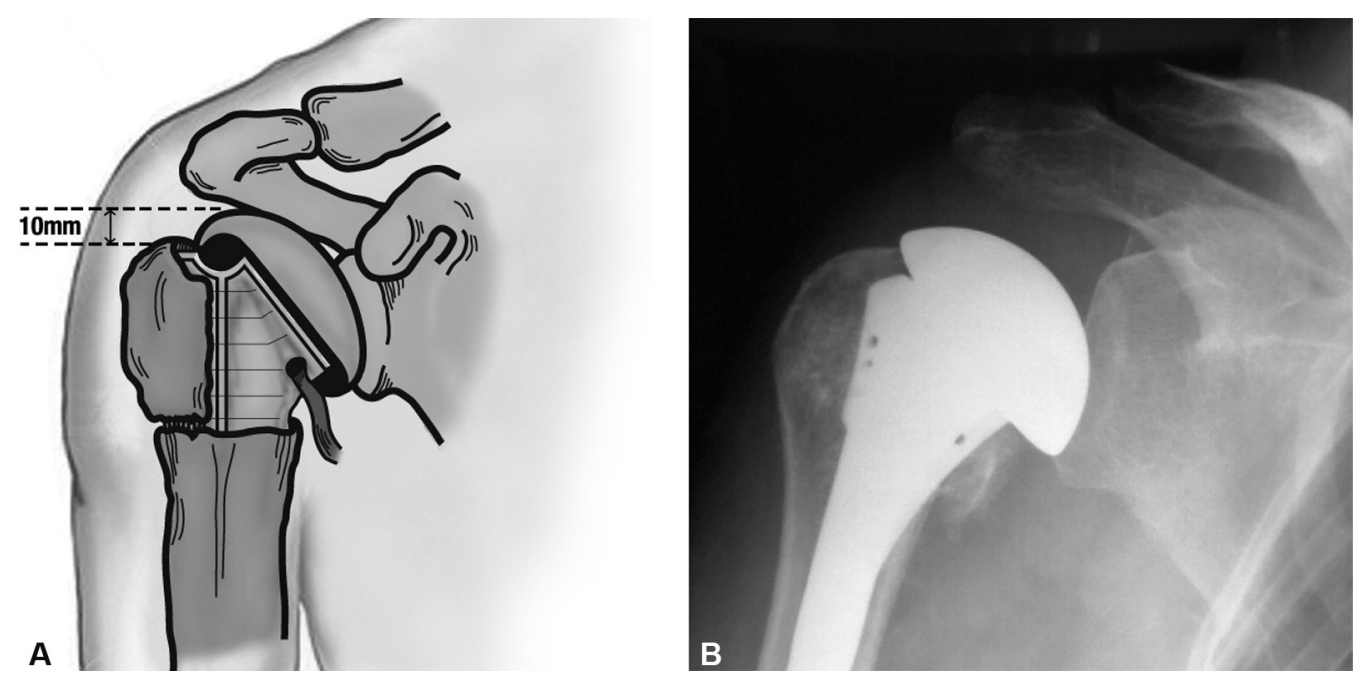

Fig. 3 (A) Esquema que demonstra a distância cabeça-tubérculo (DCT), que é uma medida objetiva da altura do tubérculo maior em relação à superfície articular da cabeça umeral da prótese, sendo em média de $8 \mathrm{~mm}$. (B) Imagem radiográfica que ilustra uma DCT dentro dos limites da normalidade.

média é de $8 \mathrm{~mm}$, com variação de $\pm 3 \mathrm{~mm}$. Distâncias superiores a $15 \mathrm{~mm}$ sugerem alto risco de complicações e maus resultados funcionais. ${ }^{7,15}$ No plano horizontal, deve-se evitar o posicionamento posteriorizado do tubérculo maior, o que aumenta o risco de falha da fixação, e, consequentemente, gera limitação da mobilidade ${ }^{8}$ (—Fig. 3A-B).

Em 2013, a revisão sistemática ${ }^{30}$ dos resultados da HA utilizando uma haste específica para fraturas comparada à ARO para o tratamento de fraturas da extremidade proximal do úmero foi publicada pela primeira vez, e teve como objetivo apresentar um resumo da melhor evidência disponível na literatura à época. Quatorze trabalhos preencheram os critérios de inclusão nessa revisão sistemática. Esforços foram empreendidos para determinar os critérios demográficos associados à utilização de cada uma das artroplastias, e seus resultados subjetivos, objetivos e radiográficos. ${ }^{30} \mathrm{~A}$ HA foi utilizada principalmente em pacientes do sexo masculino, mais jovens, e que apresentavam fraturas em quatro partes. No grupo de pacientes mais idosos que apresentam uma incidência maior de rupturas do manguito rotador associadas, estaria indicada a artroplastia reversa. ${ }^{30}$ Os resultados funcionais das HAs e AROs foram semelhantes relativos aos escores de Constant-Murley, da American Shoulder and Elbow Surgeons (ASES), assim como aos parâmetros relacionados ao exame físico. Além disso, avaliou-se a incidência de complicações e reoperações. A frequência de intercorrências clínicas foi quatro vezes maior nas AROs, o que é uma consideração marcante. As alterações radiográficas ocorreram em $90 \%$ das AROs e em $27 \%$ das HAs, apesar de parte dessas alterações, principalmente o impacto escapular inferior (notching) e a ossificação heterotópica, terem repercussão clínica discutível. Por outro lado, o percentual de reoperações entre os dois grupos não diferiu de forma significativa. ${ }^{30}$

Sebastiá-Forcada et al. ${ }^{19}$ publicaram em 2014 os resultados de um grupo randomizado e controlado comparando HA e ARO, no qual foram evidenciadas pontuações melhores no escore de Constant-Murley, no questionário Disabilities of the
Arm, Shoulder and Hand (DASH), e e no University of California at Los Angeles Shoulder Score (UCLA), e melhores flexão anterior e abdução para as AROs, sem existir diferença na rotação lateral. A fixação dos tubérculos foi realizada utilizando-se a mesma técnica para os dois grupos, e a consolidação ocorreu com maior frequência na ARO. Baseados nesses parâmetros, os autores sugeriram que a ARO seria uma melhor opção.

Em duas metanálises semelhantes, Shukla et al. ${ }^{20}$ e Wang et al. ${ }^{21}$ compararam os resultados de HAs e AROs no tratamento de fraturas da extremidade proximal do úmero. Os autores concluíram que a literatura disponível sugere que a ARO leva a resultados clínicos mais favoráveis do que a HA, com menor percentual de complicações, maiores escores da ASES, maior consolidação dos tubérculos, e melhor mobilidade de flexão anterior.

Um estudo retrospectivo da Société Française de Chirurgie Orthopédique et Traumatologique (SOFCOT) ${ }^{22}$ que comparou os resultados de HAs com AROs em fraturas da extremidade proximal do úmero, com um seguimento médio de 39 meses, demonstrou que o grupo submetido à ARO apresentou um escore de Constant-Murley ajustado superior. Por outro lado, em relação ao escore de Constant-Murley absoluto, ao DASH e ao Subjective Shoulder Value (SSV), não existiram diferenças significativas. A flexão anterior foi superior no grupo da ARO, ao passo que a rotação lateral foi melhor no grupo das HAs. Porém, em relação à rotação medial, não existiram diferenças. As complicações foram mais frequentes no grupo das HAs. O percentual de consolidação radiográfica do tubérculo maior foi igual nos 2 grupos, e ocorreu em 70\% dos casos, e o impacto inferior escapular, ou notching, ocorreu em $23 \%$ dos casos do grupo das AROs.

Gallinet et al. ${ }^{23}$ lideraram uma revisão sistemática da literatura em nome da SOFCOT, e identificaram estudos que relatavam a avaliação comparativa das HAs com as AROs para o tratamento das fraturas da extremidade proximal do úmero em pacientes com mais de 65 anos operados 
entre 2006 e 2016. Foram identificados 67 estudos, e selecionados 22 por seu nível de evidência. No grupo das AROs, houve melhor flexão anterior e abdução ativas, assim como um escore de Constant-Murley melhor. Por outro lado, as rotações lateral e medial foram inferiores no grupo das AROs. A fixação e a consolidação dos tubérculos em volta da ARO se relacionaram à capacidade de realizar as rotações. Nos casos em que não ocorreu a consolidação dos tubérculos, os resultados funcionais foram satisfatórios nas AROs, mas não nas HAs, em razão do déficit funcional marcante que existiu nesse grupo de pacientes. No grupo das AROs, a idade não foi um fator que influenciou a consolidação dos tubérculos, sendo o oposto nas HAs. As complicações foram mais frequentes no grupo das AROs, apesar de o índice de reoperação ter sido igual com relação às duas técnicas. Já a incidência de revisões, que necessitaram de troca dos implantes, foi inferior no grupo das AROs. ${ }^{23}$

Chen et al. ${ }^{24}$ realizaram em 2016 uma metanálise em rede, ou network meta-analysis (NMA), uma nova técnica que permite definir a evidência em disciplinas médicas comparando os benefícios relativos associados a múltiplas intervenções, estabelecendo, desta forma, a hierarquia de intervenções entre as várias opções de tratamento. Eles avaliaram a eficácia e a segurança da RAFI, da ARO, da HA, da fixação intramedular (FIM), e do tratamento incruento de fraturas desviadas da extremidade proximal do úmero. Utilizando essa metodologia, foram incluídos 34 trabalhos que envolviam 2.165 pacientes. $O$ grupo das ARO apresentou o maior escore de Constant-Murley e o menor percentual de complicações quando comparado ao da RAFI, HA e IM. Além disso, apresentou uma incidência menor de cirurgias adicionais do que o grupo da RAFI e da FIM. Os autores concluíram que o grupo da ARO apresentou a maior probabilidade de melhoria dos resultados funcionais e redução da incidência de complicações e necessidade de cirurgia adicional. $^{24}$

Em 2017, Du et al. ${ }^{25}$ identificaram 7 estudos randomizados a partir da pesquisa em 3 bancos de dados eletrônicos, totalizando 347 pacientes idosos submetidos ao tratamento de fraturas em 3 e 4 partes da extremidade proximal do úmero. Os pacientes foram tratados incruentamente, por RAFI, HA e ARO. O melhor escore de Constant-Murley e o menor número de reoperações ocorreram no grupo das AROs. Por outro lado, nesse grupo de pacientes idosos, a eficácia da RAFI foi a pior. A hierarquização para o escore de Constant-Murley foi ARO, HA, tratamento incruento, e RAFI. Já em relação às reoperações, foi ARO, tratamento incruento, HA e RAFI ${ }^{23}$ (- Tabela 1).

\section{Fatores que influenciam os resultados}

A utilização de uma haste umeral dedicada para o tratamento de fraturas são os implantes de escolha para a realização de uma HA ou ARO. 5,6,13,14,36,37

O surgimento dessas hastes com desenhos mais adequados à redução e à fixação dos tubérculos buscou favorecer a sua consolidação, por meio de componentes de menor perfil em sua região proximal, presença de orifícios na haste para passagem de fios de sutura resistentes, e espaço para colocação de enxerto ósseo em volume adequado para estímulo biológico à consolidação ${ }^{7,8,15,16,38,39}$ (- Fig. 4A-B).

Tabela 1 Dados clínicos de estudos comparativos entre a ARO e a HA

\begin{tabular}{|c|c|c|c|c|c|c|c|}
\hline Autor (ano) & Amostra & $\begin{array}{l}\text { Escore de } \\
\text { Constant_Murley }\end{array}$ & FA & Abd & $\mathrm{RL}$ & RM & $\begin{array}{l}\text { Consolidação do } \\
\text { tubérculo maior }\end{array}$ \\
\hline $\begin{array}{l}\text { Cuff e Pupello }{ }^{26} \\
(2013)\end{array}$ & $\begin{array}{l}27 \text { ARO } \\
26 \mathrm{HA}\end{array}$ & $\mathrm{NI}$ & $\begin{array}{l}139^{\circ} \mathrm{ARO} \\
100^{\circ} \mathrm{HA}\end{array}$ & $\mathrm{NI}$ & $\begin{array}{l}24^{\circ} \mathrm{ARO} \\
25^{\circ} \mathrm{HA}\end{array}$ & $\mathrm{NI}$ & $\begin{array}{l}\text { ARO } 83 \% \\
\text { HA } 37 \% \\
(p=0,17)\end{array}$ \\
\hline $\begin{array}{l}\text { Namdari } \\
\text { et al. }{ }^{30}(2013)\end{array}$ & $\begin{array}{l}210 \mathrm{ARO} \\
231 \mathrm{HA}\end{array}$ & $\begin{array}{l}50,3 \text { ARO } \\
(41,1-70,9) \\
56 \mathrm{HA} \\
(38,7-61,9)\end{array}$ & $\begin{array}{l}114^{\circ} \mathrm{ARO} \\
\left(97^{\circ}-137^{\circ}\right) \\
117^{\circ} \mathrm{HA} \\
\left(96^{\circ}-133^{\circ}\right)\end{array}$ & $\begin{array}{l}92^{\circ} \text { ARO } \\
\left(66^{\circ}-119^{\circ}\right) \\
111^{\circ} \mathrm{HA} \\
\left(70^{\circ}-151^{\circ}\right)\end{array}$ & $\begin{array}{l}20^{\circ} \mathrm{ARO} \\
\left(3^{\circ}-37^{\circ}\right) \\
34^{\circ} \mathrm{HA} \\
\left(21^{\circ}-47^{\circ}\right)\end{array}$ & $\mathrm{NI}$ & $\mathrm{NI}$ \\
\hline $\begin{array}{l}\text { Mata-Fink et al. }{ }^{27} \\
(2013)\end{array}$ & $\begin{array}{l}377 \mathrm{ARO} \\
504 \mathrm{HA}\end{array}$ & $\begin{array}{l}\text { 54,7 ARO } \\
48,6 \mathrm{HA}\end{array}$ & $\begin{array}{l}113^{\circ} \mathrm{ARO} \\
92^{\circ} \mathrm{HA}\end{array}$ & $\mathrm{NI}$ & $\begin{array}{l}20^{\circ} \mathrm{ARO} \\
22^{\circ} \mathrm{HA}\end{array}$ & $\begin{array}{l}\text { L3 ARO } \\
\text { T12 HA }\end{array}$ & $\mathrm{NI}$ \\
\hline $\begin{array}{l}\text { Sebastiá-Forcada } \\
\text { et al. }{ }^{19}(2014)\end{array}$ & $\begin{array}{l}31 \mathrm{ARO} \\
31 \mathrm{HA}\end{array}$ & $\begin{array}{l}56,1 \text { ARO } \\
(24-80) \\
40 \mathrm{HA} \\
(8-74)\end{array}$ & $\begin{array}{l}120^{\circ} \mathrm{ARO} \\
\left(40^{\circ}-180^{\circ}\right) \\
80^{\circ} \mathrm{HA} \\
\left(20^{\circ}-180^{\circ}\right)\end{array}$ & $\begin{array}{l}113^{\circ} \text { ARO } \\
\left(50^{\circ}-170^{\circ}\right) \\
79^{\circ} \mathrm{HA} \\
\left(30^{\circ}-150^{\circ}\right)\end{array}$ & $\begin{array}{l}30^{\circ} \text { ARO } \\
\left(0^{\circ}-10^{\circ}\right) \\
26^{\circ} \mathrm{HA} \\
\left(0^{\circ}-10^{\circ}\right)\end{array}$ & $\begin{array}{l}\text { Sacro ARO } \\
\text { Sacro HA }\end{array}$ & $\begin{array}{l}\text { ARO } 64,5 \% \\
\text { HA 56,7\% } \\
(p=N I)\end{array}$ \\
\hline $\begin{array}{l}\text { Baudi et al. }{ }^{28} \\
(2014)\end{array}$ & $\begin{array}{l}25 \text { ARO } \\
28 \text { HA }\end{array}$ & $\begin{array}{l}\text { 56,2 ARO } \\
42,3 \mathrm{HA}\end{array}$ & $\begin{array}{l}131^{\circ} \mathrm{ARO} \\
89^{\circ} \mathrm{HA}\end{array}$ & $\begin{array}{l}128^{\circ} \mathrm{ARO} \\
82^{\circ} \mathrm{HA}\end{array}$ & $\begin{array}{l}15^{\circ} \mathrm{ARO} \\
23^{\circ} \mathrm{HA}\end{array}$ & $\mathrm{NI}$ & $\begin{array}{l}\text { ARO 84\% } \\
\text { HA 37\% } \\
(p<0,05)\end{array}$ \\
\hline $\begin{array}{l}\text { Ferrel et al. }{ }^{29} \\
(2015)\end{array}$ & $\begin{array}{l}322 \mathrm{ARO} \\
1.024 \mathrm{HA}\end{array}$ & $\begin{array}{l}54,6 \text { ARO } \\
58 \text { HA }\end{array}$ & $\begin{array}{l}118^{\circ} \mathrm{ARO} \\
108^{\circ} \mathrm{HA}\end{array}$ & $\begin{array}{l}98^{\circ} \mathrm{ARO} \\
94^{\circ} \mathrm{HA}\end{array}$ & $\begin{array}{l}20^{\circ} \mathrm{ARO} \\
30^{\circ} \mathrm{HA}\end{array}$ & $\mathrm{NI}$ & $\begin{array}{l}\text { ARO } 82,8 \% \\
\text { HA } 73,9 \%\end{array}$ \\
\hline $\begin{array}{l}\text { Bonnevialle } \\
\text { et al. }{ }^{22}(2016)\end{array}$ & $\begin{array}{l}41 \mathrm{ARO} \\
57 \mathrm{HA}\end{array}$ & $\begin{array}{l}57 \text { ARO } \\
(23-90) \\
54 \mathrm{HA} \\
(19-89)\end{array}$ & $\begin{array}{l}130^{\circ} \mathrm{ARO} \\
\left(50^{\circ}-180^{\circ}\right) \\
112^{\circ} \mathrm{HA} \\
\left(20^{\circ}-180^{\circ}\right)\end{array}$ & $\mathrm{NI}$ & $\begin{array}{l}23^{\circ} \mathrm{ARO} \\
\left(-20^{\circ}-70^{\circ}\right) \\
28^{\circ} \mathrm{HA} \\
\left(0^{\circ}-80^{\circ}\right)\end{array}$ & $\begin{array}{l}\text { Sacro ARO } \\
\text { L3 HA }\end{array}$ & $\begin{array}{l}\text { ARO 73\% } \\
\text { HA 72\% } \\
(p=0,95)\end{array}$ \\
\hline
\end{tabular}

Abreviaturas: Abd, abdução; ARO, artroplastia reversa do ombro; FA, flexão anterior; HA, hemiartroplastia; NI, não informado; RL, rotação lateral; $\mathrm{RM}$, rotação medial. 

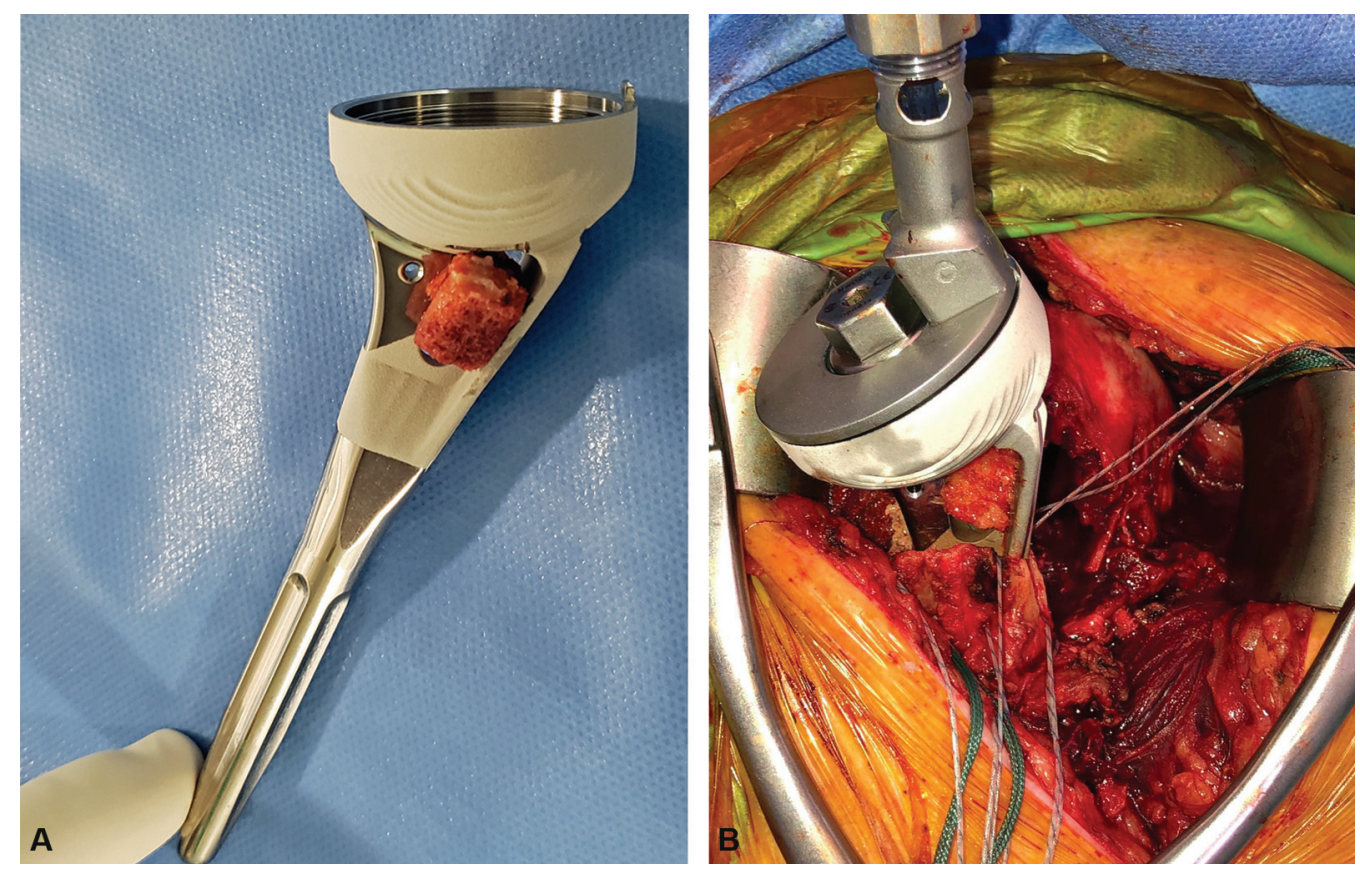

Fig. 4 (A) Imagem da haste de fratura com enxerto ósseo no orifício específico. (B) Imagem perioperatória de uma haste específica para o tratamento de fratura.

Apesar de toda a evolução tecnológica, a HA persiste apresentando resultados funcionais heterogêneos, com complicações relacionadas à consolidação dos tubérculos $7,8,40$ (-Figs. 5 e 6 ).

Provavelmente, devido a dificuldades técnicas para a realização de uma HA, as AROs estão se tornando o implante de escolha para o tratamento cirúrgico dessas lesões. O que merece discussão é determinar se, mesmo nas AROs, o uso de

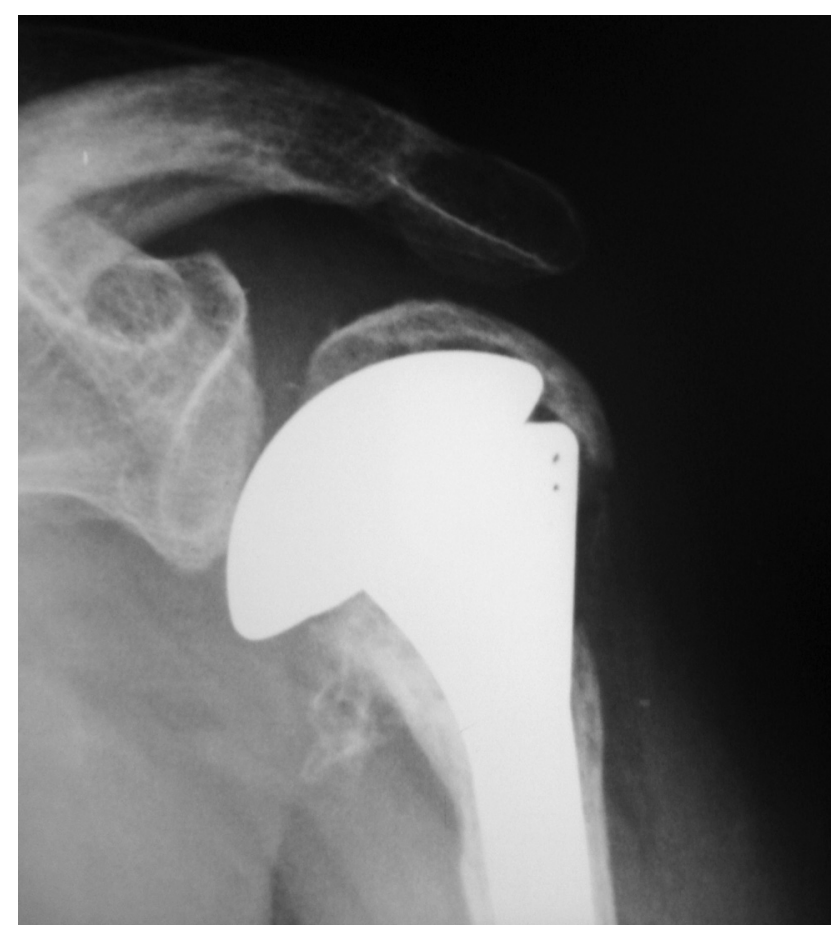

Fig. 5 Imagem radiográfica pós-operatória de hemiartroplastia com avulsão do tubérculo maior. desenhos de implantes específicos para o tratamento de fraturas complexas da extremidade proximal do úmero é necessário, ou se desenhos comuns de implantes, já utilizados no tratamento de outras doenças, são suficientes. ${ }^{38,39}$

Nas AROs, aparentemente, o uso de componentes umerais de baixo perfil proximal, medialização da epífise da haste, área medial do colo da haste lisa e polida, e a parte lateral da metáfise com hidroxiapatita favorecem a redução e a

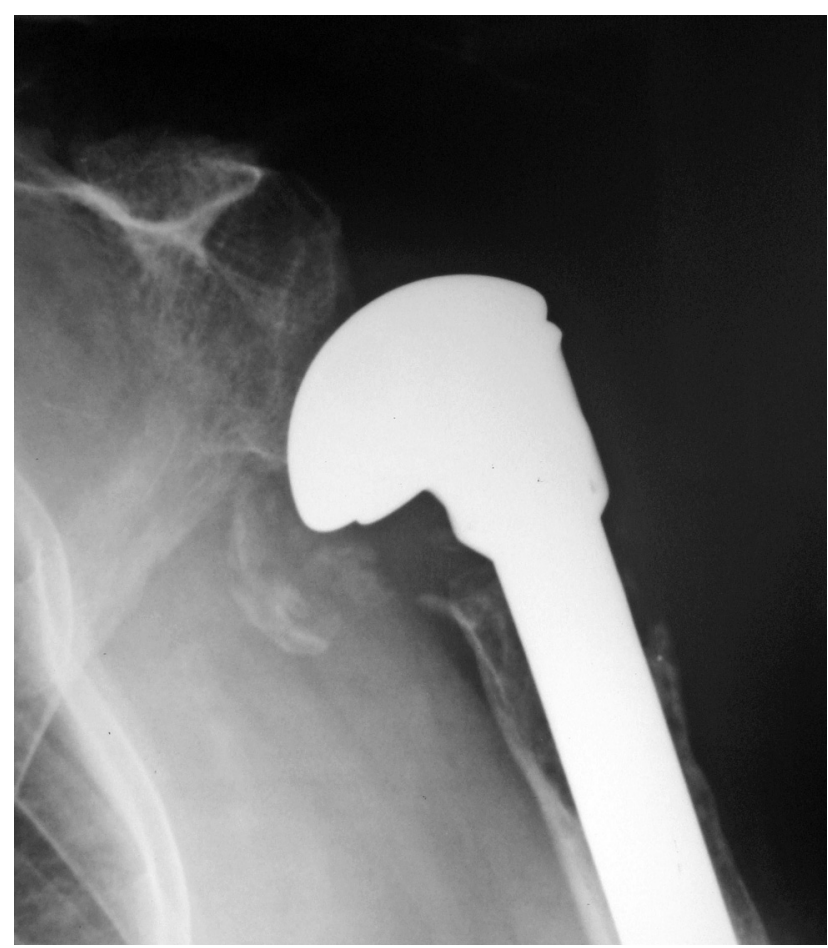

Fig. 6 Imagem radiográfica pós-operatória de hemiartroplastia com avulsão do tubérculo menor. 
estabilização dos tubérculos, além do aumento percentual de consolidação, influenciando a mobilidade rotacional pósoperatória.. ${ }^{4,37}$ A posição do polietileno inlay em relação à haste também aparentemente contribui para a maior integração dos tubérculos. ${ }^{38,39}$

Além disso, para a realização de uma ARO, existem no mercado implantes com desenhos que permitem lateralizar ou medializar o centro de rotação da articulação do ombro. Um estudos comparativo ${ }^{38}$ sugere que a lateralização do centro de rotação da articulação e um menor ângulo cervico-diafisário melhoram a função dos músculos que atuam na rotação externa do ombro, melhorando os resultados clínicos quanto à mobilidade de rotação lateral nas AROs. Por outro lado, Verdano et al. ${ }^{39}$ realizaram, em 2018, uma avaliação retrospectiva dos resultados clínicos e radiográficos, comparando próteses reversas lateralizadas no lado umeral com o modelo medializado tipo Grammont para o tratamento das fraturas desviadas da extremidade proximal do úmero. O escore de Constant-Murley e as amplitudes de rotação lateral e medial foram similares, ao passo que a flexão anterior e a consolidação do tubérculo maior ocorreram em um maior percentual de pacientes do grupo com prótese do tipo medializada. Porém, nesse estudo ${ }^{39}$ não foi utilizado nenhum tipo de componente umeral com desenho específico para o tratamento de fraturas. Esse trabalho, com as limitações próprias desse tipo de revisão, não esclarece qual deveria ser o implante a ser utilizado nos pacientes. ${ }^{39-41}$

A ARO para o tratamento de fraturas é tradicionalmente realizada com componentes umerais cimentados. A literatura sugere que componentes umerais não cimentados nas AROs para o tratamento de fraturas apresentam resultados inferiores de acordo com os escores subjetivos, apesar de não existir correlação com a dor, mobilidade e consolidação do tubérculo. $^{42}$

Um ponto-chave na realização das AROs é o preparo e a colocação do componente da glenoide, mesmo no contexto das fraturas da extremidade proximal do úmero, apesar de poucos estudos abordarem esse tema. Há relatos referentes a afrouxamento da base metálica $(0,52 \%$ a $3,5 \%)$ e alta incidência de impacto escapular $(43,6 \%)$ relacionados a posicionamento inadequado e versão incorreta da glenosfera. São considerados fatores de prevenção do impacto escapular: inclinação e posicionamento inferior do componente da glenoide, ângulo cérvico-diafisário menor do que $155^{\circ}$, e implantes lateralizados. Sendo assim, além da seleção do implante, a compreensão da morfologia da glenoide também é um fator importante. ${ }^{39}$

Em relação ao momento em que os pacientes devem ser operados, a literatura relata bons resultados da utilização da ARO tanto para o tratamento das fraturas agudas desviadas quanto para as fraturas tratadas tardiamente. ${ }^{36,37}$

Vale ressaltar a importância do conceito de "tardio" na literatura. Após 20 dias, a mobilização dos tubérculos e a fixação é comprometida pela consolidação e reabsorção óssea. ${ }^{43} \mathrm{Na}$ opinião pessoal dos autores deste estudo, o tratamento de sequelas das fraturas proximais do úmero implicam uma dissecção mais complexa e extensa, e é necessário estar preparado para lidar com o comprometi- mento do estoque ósseo que poderá existir dependendo das características da lesão. Além disso, é esperado que ocorra uma maior perda sanguínea, com suas eventuais consequências, e maior percentual de complicações neurológicas, instabilidade, e infecção.

Dezfuli et al. ${ }^{43}$ avaliaram 49 pacientes que foram submetidos a ARO para tratamento de fraturas proximais do úmero. Os autores, estratificaram os pacientes em fraturas agudas e crônicas operadas por diferentes razões. 0 grupo de fraturas agudas apresentou resultados superiores a todos os outros. Nos casos de fraturas envelhecidas ou sequelas de fraturas, como consolidações viciosas e pseudoartroses, os resultados das AROs, apesar de inferiores aos de AROs em fraturas agudas, foram superiores aos das AROs realizadas para revisão de resultados ruins em pacientes com fraturas primariamente operados por outras técnicas, como HA ou osteossíntese.

Seidl et al. ${ }^{37}$ compararam pacientes submetidos a ARO para tratamento de fraturas agudas ocorridas no período de até quatro semanas com outro grupo, em que os pacientes foram submetidos previamente a outro tipo de tratamento. Os autores concluíram que, apesar de os pacientes agudos e secundários poderem apresentar resultados satisfatórios, o grupo de agudos apresentou resultados superiores em relação à consolidação dos tubérculos, e uma melhor rotação externa.

Em 2019, Torchia et al. ${ }^{36}$ realizaram uma metanálise que incluiu 16 estudos com 322 pacientes para determinar se os pacientes deveriam ser operados na fase aguda ou tardiamente. Desses, 4 eram estudos comparativos, e 12 eram séries de casos. No grupo de estudos comparativos, não foram evidenciadas diferenças na flexão anterior, nos escores clínicos, ou nas reoperações. No grupo de pacientes tratados tardiamente, ocorreu ganho de mais $6^{\circ}$ graus de rotação lateral, o que foi estatisticamente significativo. Os autores concluíram que, levando em conta o risco associado à cirurgia na população idosa, deve-se tentar o tratamento incruento, reservando a ARO para os casos em que essa terapêutica falhar. ${ }^{36}$

Boileau et al. ${ }^{34}$ estabeleceram a hipótese de que fixar o tubérculo maior e a sua consolidação iriam produzir melhores resultados clínicos nos pacientes submetidos a ARO para tratamento de fratura proximal do úmero. Pacientes com fraturas e com idade média de 80,4 anos foram submetidos a artroplastia reversa, utilizando uma haste específica para fraturas, o que permite a colocação de enxerto ósseo retirado da cabeça humeral e a sutura dos tubérculos ao seu redor. A consolidação das fraturas ocorreu em $84 \%$ dos pacientes, com 4 casos de reabsorção e 2 de não união e migração. Esse grupo de 6 pacientes apresentou um SSV de 65\%, contra $83 \%$ no grupo em que houve a consolidação dos tubérculos. Em relação à amplitude de movimentos, a flexão anterior foi de $115^{\circ}$, contra $141^{\circ}$, e rotação lateral de $11^{\circ}$ e $27^{\circ}$, respectivamente. Apesar da idade avançada dos pacientes, os autores concluíram que a reconstrução e a consolidação dos tubérculos proporcionam um melhor resultado clínico, assim como melhores flexão anterior e rotação lateral. ${ }^{34}$ Cabe chamar a atenção para o número não expressivo de pacientes que sustenta essa conclusão. 


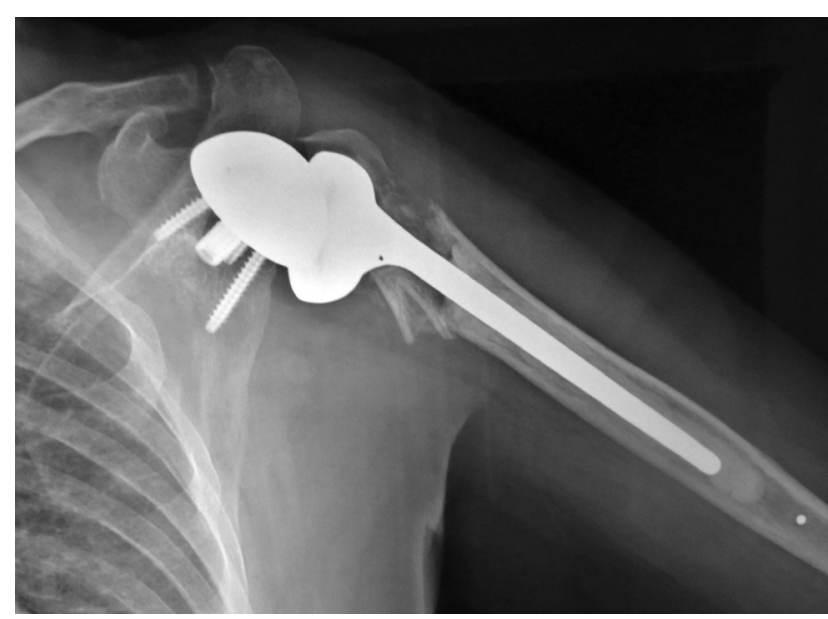

Fig. 7 Imagem radiográfica que ilustra a consolidação do tubérculo maior com migração proximal associada a uma artroplastia reversa do ombro (ARO).

A experiência da SOFCOT demonstra que a fixação e a consolidação dos tubérculos em volta da ARO corroboram a expectativa de uma melhor habilidade de realizar a rotação. ${ }^{35}$

Em 2018, Torrens et al. ${ }^{33}$ realizaram um estudo retrospectivo de 41 casos consecutivos para avaliar a influência da consolidação do tubérculo maior nos resultados funcionais, após a realização de uma ARO para tratamento de fraturas proximais do úmero. A consolidação ocorreu em $68 \%$ dos casos. Neste estudo, foram evidenciados escores funcionais, amplitude de movimentos, e impacto escapular iguais tanto no grupo em que ocorreu a consolidação quanto naquele em que não houve consolidação. Apesar da expectativa da obtenção de melhores resultados em relação à função do ombro, isso não foi demonstrado nessa série de casos. ${ }^{33}$ (-Figs. 7 e $\mathbf{8 A - C}$ ).

É possível concluir que a consolidação dos tubérculos associada à realização da artroplastia reversa para o tratamento de fraturas proximais do úmero apresenta resultados controversos na literatura acerca de sua influência nos resultados clínicos e na amplitude de movimentos, especial- mente nas rotações lateral e medial fundamentais às atividades cotidianas (- Fig. 9A-B). ${ }^{32-34}$

Vale ressaltar algumas diferenças de resultados entre as AROs e as HAs no tratamento das fraturas da extremidade proximal do úmero, no que se relaciona à consolidação dos tubérculos. Na ARO, mesmo quando não ocorre a consolidação dos tubérculos, os resultados funcionais podem ser satisfatórios, diferente da HA, na qual ocorre um déficit funcional marcante. ${ }^{32-34}$

A realização da artroplastia do ombro não está associada a sangramento de grande volume, mas cuidados que permitam diminuir a perda sanguínea devem ser tomados. O ácido tranexâmico (ATX), que é um agente antifibrinolítico, tem potencial de redução na perda sanguínea e na necessidade de transfusão sanguínea após artroplastias do quadril e joelho. ${ }^{44}$

Diversos trabalhos ${ }^{44-46}$ foram realizados com o objetivo de avaliar a eficácia e segurança do uso do ATX. Gillespie et al. ${ }^{44}$ realizaram trabalho randomizado envolvendo 111 pacientes divididos em 2 grupos. O que recebeu a droga, 2 gramas de ATX por aplicação tópica na ferida operatória, em comparação ao que recebeu um placebo, apresentou menor perda sanguínea, assim como menor redução sérica de hemoglobina.

Kirsch et al. ${ }^{45}$ e Sun et al. ${ }^{46}$ realizaram metanálises do uso do ATX em artroplastia do ombro, e os resultados demonstraram uma redução significativa na alteração da hemoglobina, o que sugere uma redução da necessidade de transfusão em suas revisões.

Dessa forma, a literatura parece clara em sugerir a utilização do ATX em associação com a realização das artroplastias do ombro.

\section{Complicações}

A média geral de complicações das AROs nas fraturas agudas é de $25 \%$, sendo $17,4 \%$ classificadas como menores, e 7,6\%, maiores. ${ }^{47,48}$

Lopiz et al. ${ }^{49}$ realizaram uma avaliação retrospectiva de 42 pacientes submetidos a ARO para o tratamento de fraturas da extremidade proximal do úmero divididos em 2 grupos: um com pacientes com idade menor do que 80 anos $(<80)$ e
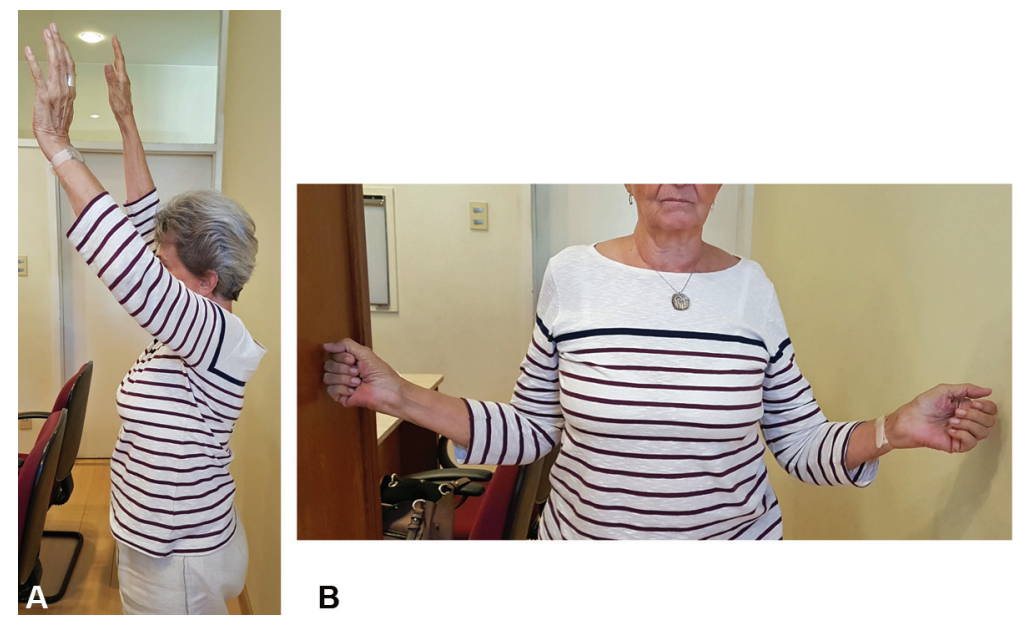
B

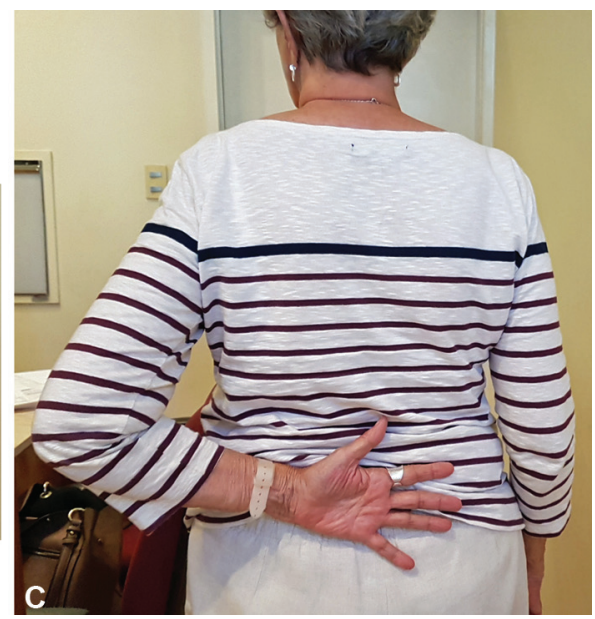

Fig. 8 Amplitude de movimentos do paciente descrito na figura anterior. A amplitude de movimentos foi conseguida apesar do aspecto radiográfico. (A) - flexão anterior; (B) - rotação lateral; (C) - rotação medial. 

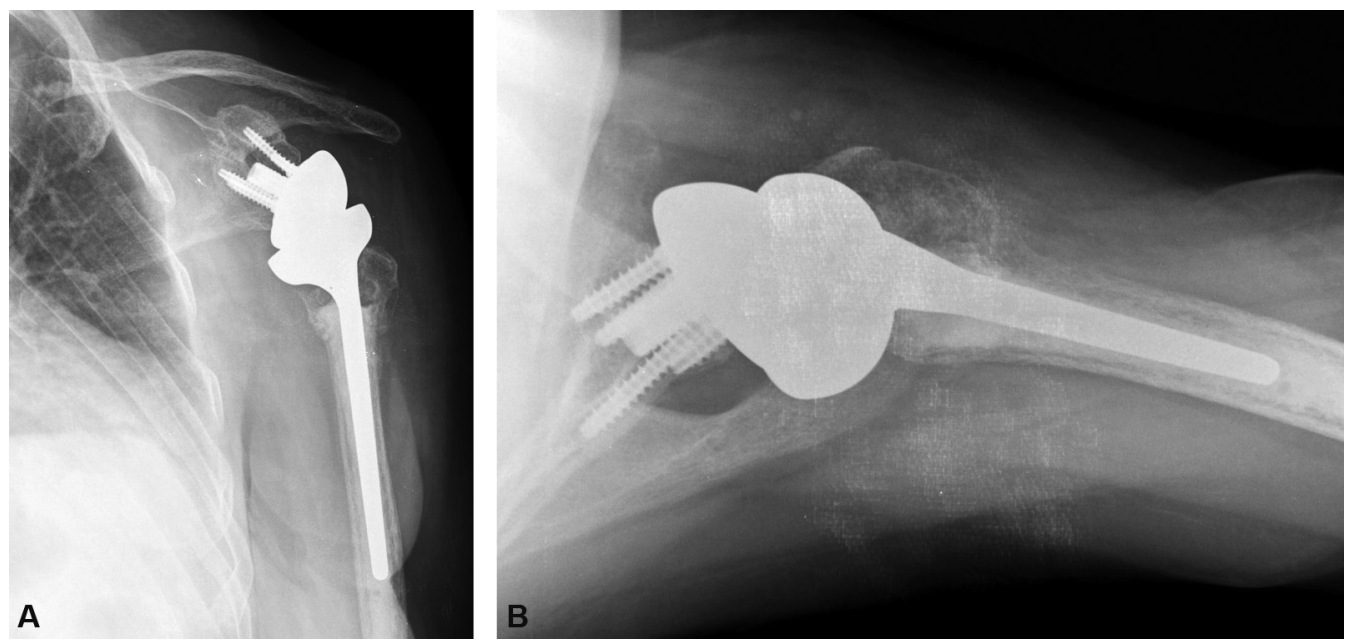

Fig. 9 (A) Imagem radiográfica em incidência anteroposterior de ARO com o tubérculo maior consolidado, porém, não reproduzindo a anatomia normal. (B) Imagem em incidência axilar.

outro com idade igual ou maior do que $80(=80)$, com seguimento médio de 32,6 meses. O escore de ConstantMurley, ajustado para o sexo e a idade, foi de 64 para o grupo $<80$, e de 33 para $0=80$. Em relação à amplitude de movimentos, os resultados foram, respectivamente para os grupos $<80$ e $\geq 80$ : flexão anterior de $126^{\circ}$ e $110^{\circ}$; abdução de $117^{\circ}$ e $105^{\circ}$; rotação lateral de $22^{\circ}$ e $20^{\circ}$; e rotação medial de L3 e sacro. Ocorreram 9,5\% de complicações relacionadas à prótese e ao procedimento nos 2 grupos. Os autores concluíram que a idade é um fator crítico para o sucesso da ARO. $^{49}$

Noguera et al. ${ }^{47}$ avaliaram as complicações associadas à utilização da ARO para tratamento das fraturas proximais do úmero em 103 pacientes com idade superior a 65 anos, com média de 77,3 anos. O percentual total de complicações foi de $25 \%$, sendo $17,4 \%$ consideradas simples, e $7,6 \%$, severas. A correlação de complicações graves e o escore da American Society of Anesthesiologists (ASA) foi estatisticamente significativa, sendo que a ocorrência das complicações se deu num período de cerca de noventa dias. Também foi evidenciada uma tendência a complicações simples durante a primeira semana, associada a um ASA alto. Doença reumatoide foi significantemente associada a complicações graves. O percentual de transfusão foi de $11.5 \%$. A conclusão do estudo foi a de que a média de complicações graves nos pacientes submetidos a ARO para fraturas foi baixa na população idosa. 47

Em uma revisão sistemática e metanálise, Austin et al. ${ }^{50}$ sugerem que a ARO resulta em melhora da amplitude de movimentos, dos escores de acompanhamento clínico, das taxas de reoperações de todas as causas, e na ausência de diferença nas taxas de infecção quando comparada à HA. Os autores concluem que o uso da ARO para o tratamento das fraturas agudas da extremidade proximal do úmero na população idosa pode levar a um melhor resultado no curto e médio prazos em comparação à HA. Por fim, eles sugerem que avaliações de longo prazo são necessárias para definir se esses benefícios das AROs irão persistir. ${ }^{50}$

\section{Custos}

O aumento significativo da complexidade do cenário dos sistemas de saúde no mundo necessita de racionalização dos custos e gastos. ${ }^{51}$ A viabilização econômica da incorporação de tecnologias de alto custo depende da interpretação dos resultados clínicos no contexto da compensação dos custos. $^{51} \mathrm{O}$ aumento do uso das AROs no tratamento das fraturas da extremidade proximal do úmero torna essa questão extremamente relevante, principalmente porque o uso da ARO nessas situações se associa a internações mais prolongadas, maior incidência de complicações, e custos mais altos em comparação ao uso da ARO no tratamento da artropatia do manguito rotador. ${ }^{52}$ Estudos relevantes devem considerar a relação entre os custos hospitalares totais, como: tempo de hospitalização, tempo cirúrgico, taxa de hemotransfusão, complicações clínicas e cirúrgicas, custos dos implantes, e compará-los aos resultados clínicos funcionais. ${ }^{52-54}$

Liu et al. ${ }^{52}$ demonstraram que as AROs têm custos superiores às HAs, mas sem diferença no tempo de internação, no uso de hemoderivados, e na amplitude de movimentos final, apesar dos melhores escores de dor e função. ${ }^{52}$ Os autores sugeriram que uma investigação mais aprofundada quanto aos custos da reabilitação pós-operatória, cuidados especiais de enfermagem, e taxa de revisão cirúrgica são necessários para a melhor interpretação do custo-benefício desse tipo de tratamento. $^{52}$

Algumas análises financeiras sugerem que a ARO no tratamento da fratura da extremidade proximal do úmero em idosos é a estratégia preferida quando comparada à HA. ${ }^{53,54}$ Cálculos de custos demonstram que o incremento do custo da ARO no tratamento dessas fraturas encontra-se bem abaixo dos limiares padrões de pagamento por tecnologias que melhoram a qualidade de vida, sendo seu custobenefício similar ao de outras terapêuticas cirúrgicas de amplo sucesso na prática ortopédica, como as artroplastias totais de quadril no tratamento da artrose. ${ }^{53}$ 


\section{Considerações Finais}

Em virtude dos resultados heterogêneos da HA, diretamente relacionados à qualidade da redução e à consolidação dos tubérculos, a ARO tem sido utilizada com uma frequência progressiva, com resultados funcionais mais homogêneos e taxas de complicações equivalentes às da HA.

Existem dúvidas quanto à seleção de pacientes adequados a cada técnica (HA e ARO), principalmente devido às limitações inerentes a todas as metanálises, em virtude da heterogeneidade dos estudos.

\section{Conflito de Interesses}

Os autores declaram não haver conflito de interesses.

\section{Referências}

1 Kim SH, Szabo RM, Marder RA. Epidemiology of humerus fractures in the United States: nationwide emergency department sample, 2008. Arthritis Care Res (Hoboken) 2012;64(03):407-414

2 Neer CS II. Displaced proximal humeral fractures. I. Classification and evaluation. J Bone Joint Surg Am 1970;52(06):1077-1089

3 Maier D, Jaeger M, Izadpanah K, Strohm PC, Suedkamp NP. Proximal humeral fracture treatment in adults. J Bone Joint Surg Am 2014;96(03):251-261

4 Checchia SL, Doneux PS, Miyazaki AN, et al. Tratamento das fraturas do terço proximal do úmero com a prótese parcial Eccentra ${ }^{\circledR}$. Rev Bras Ortop 2005;40(03):130-140

5 Murachovsky J, Ikemoto RY, Nascimento LG, Fujiki EN, Milani C, Warner JJ. Pectoralis major tendon reference (PMT): a new method for accurate restoration of humeral length with hemiarthroplasty for fracture. J Shoulder Elbow Surg 2006;15 (06):675-678

6 Brandão BL, Amaral MV, Cohen M, et al. Osteossíntese das fraturas da extremidade proximal do úmero com sistema de placa de ângulo fixo com parafusos bloqueados: técnica e resultados. Rev Bras Ortop 2009;44(02):106-111

7 Boileau P, Winter M, Cikes A, et al. Can surgeons predict what makes a good hemiarthroplasty for fracture? J Shoulder Elbow Surg 2013;22(11):1495-1506

8 Cadet ER, Ahmad CS. Hemiarthroplasty for three- and four-part proximal humerus fractures. J Am Acad Orthop Surg 2012;20(01): $17-27$

9 Robinson CM, Page RS, Hill RM, Sanders DL, Court-Brown CM, Wakefield AE. Primary hemiarthroplasty for treatment of proximal humeral fractures. J Bone Joint Surg Am 2003;85(07): 1215-1223

10 Mighell MA, Kolm GP, Collinge CA, Frankle MA. Outcomes of hemiarthroplasty for fractures of the proximal humerus. J Shoulder Elbow Surg 2003;12(06):569-577

11 Bufquin T, Hersan A, Hubert L, Massin P. Reverse shoulder arthroplasty for the treatment of three- and four-part fractures of the proximal humerus in the elderly: a prospective review of 43 cases with a short-term follow-up. J Bone Joint Surg Br 2007;89 (04):516-520

12 Dillon MT, Prentice HA, Burfeind WE, Chan PH, Navarro RA. The increasing role of reverse total shoulder arthroplasty in the treatment of proximal humerus fractures. Injury 2019;50(03): 676-680

13 Jo YH, Lee KH, Lee BG. Surgical trends in elderly patients with proximal humeral fractures in South Korea: a population-based study. BMC Musculoskelet Disord 2019;20(01):136

14 Neer CS II. Displaced proximal humeral fractures. II. Treatment of three-part and four-part displacement. J Bone Joint Surg Am 1970;52(06):1090-1103
15 Boileau P, Sinnerton RJ, Chuinard C, Walch G. Arthroplasty of the shoulder. J Bone Joint Surg Br 2006;88(05):562-575

16 Garofalo R, Flanagin B, Castagna A, Lo EY, Krishnan SG. Reverse shoulder arthroplasty for proximal humerus fracture using a dedicated stem: radiological outcomes at a minimum 2 years of follow-up-case series. J Orthop Surg Res 2015;10:129

17 Boileau P, Watkinson DJ, Hatzidakis AM, Balg F. Grammont reverse prosthesis: design, rationale, and biomechanics. J Shoulder Elbow Surg 2005;14(1, Suppl S)147S-161S

18 Gerber C, Pennington SD, Nyffeler RW. Reverse total shoulder arthroplasty. J Am Acad Orthop Surg 2009;17(05):284-295

19 Sebastiá-Forcada E, Cebrián-Gómez R, Lizaur-Utrilla A, Gil-Guillén $V$. Reverse shoulder arthroplasty versus hemiarthroplasty for acute proximal humeral fractures. A blinded, randomized, controlled, prospective study. J Shoulder Elbow Surg 2014;23(10): 1419-1426

20 Shukla DR, McAnany S, Kim J, Overley S, Parsons BO. Hemiarthroplasty versus reverse shoulder arthroplasty for treatment of proximal humeral fractures: a meta-analysis. J Shoulder Elbow Surg 2016;25(02):330-340

21 Wang J, Zhu Y, Zhang F, Chen W, Tian Y, Zhang Y. Meta-analysis suggests that reverse shoulder arthroplasty in proximal humerus fractures is a better option than hemiarthroplasty in the elderly. Int Orthop 2016;40(03):531-539

22 Bonnevialle N, Tournier C, Clavert P, Ohl X, Sirveaux F, Saragaglia Dla Société française de chirurgie orthopédique et traumatologique. Hemiarthroplasty versus reverse shoulder arthroplasty in 4part displaced fractures of the proximal humerus: Multicenter retrospective study. Orthop Traumatol Surg Res 2016;102(05): 569-573

23 Gallinet D, Ohl X, Decroocq L, Dib C, Valenti P, Boileau PFrench Society for Orthopaedic Surgery (SOFCOT). Is reverse total shoulder arthroplasty more effective than hemiarthroplasty for treating displaced proximal humerus fractures in older adults? A systematic review and meta-analysis. Orthop Traumatol Surg Res 2018;104(06):759-766

24 Chen L, Xing F, Xiang Z. Effectiveness and safety of interventions for treating adults with displaced proximal humeral fracture: A network meta-analysis and systematic review. PLoS One 2016;11 (11):e0166801

25 Du S, Ye J, Chen H, Li X, Lin Q. Interventions for Treating 3- or 4part proximal humeral fractures in elderly patient: A network meta-analysis of randomized controlled trials. Int J Surg 2017; 48:240-246

26 Cuff DJ, Pupello DR. Comparison of hemiarthroplasty and reverse shoulder arthroplasty for the treatment of proximal humeral fractures in elderly patients. J Bone Joint Surg Am 2013;95(22): 2050-2055

27 Mata-Fink A, Meinke M, Jones C, Kim B, Bell JE. Reverse shoulder arthroplasty for treatment of proximal humeral fractures in older adults: a systematic review. J Shoulder Elbow Surg 2013;22(12): 1737-1748

28 Baudi P, Campochiaro G, Serafini F, et al. Hemiarthroplasty versus reverse shoulder arthroplasty: comparative study of functional and radiological outcomes in the treatment of acute proximal humerus fracture. Musculoskelet Surg 2014;98(Suppl 1):19-25

29 Ferrel JR, Trinh TQ Fischer RA. Reverse total shoulder arthroplasty versus hemiarthroplasty for proximal humeral fractures: a systematic review. J Orthop Trauma 2015;29(01):60-68

30 Namdari S, Horneff JG, Baldwin K. Comparison of hemiarthroplasty and reverse arthroplasty for treatment of proximal humeral fractures: a systematic review. J Bone Joint Surg Am 2013;95 (18):1701-1708

31 Simovitch RW, Roche CP, Jones RB, et al. Effect of Tuberosity Healing on Clinical Outcomes in Elderly Patients Treated With a Reverse Shoulder Arthroplasty for 3- and 4-Part Proximal Humerus Fractures. J Orthop Trauma 2019;33(02):e39-e45 
32 Chun YM, Kim DS, Lee DH, Shin SJ. Reverse shoulder arthroplasty for four-part proximal humerus fracture in elderly patients: can a healed tuberosity improve the functional outcomes? J Shoulder Elbow Surg 2017;26(07):1216-1221

33 Torrens C, Alentorn-Geli E, Mingo F, Gamba C, Santana F. Reverse shoulder arthroplasty for the treatment of acute complex proximal humeral fractures: Influence of greater tuberosity healing on the functional outcomes. J Orthop Surg (Hong Kong) 2018;26(01): 2309499018760132

34 Boileau P, Alta TD, Decroocq L, et al. Reverse shoulder arthroplasty for acute fractures in the elderly: is it worth reattaching the tuberosities? J Shoulder Elbow Surg 2019;28(03):437-444

35 Ohl X, Bonnevialle N, Gallinet D, et al. SOFCOT. How the greater tuberosity affects clinical outcomes after reverse shoulder arthroplasty for proximal humeral fractures. J Shoulder Elbow Surg 2018;27(12):2139-2144

36 Torchia MT, Austin DC, Cozzolino N, Jacobowitz L, Bell JE. Acute versus delayed reverse total shoulder arthroplasty for the treatment of proximal humeral fractures in the elderly population: a systematic review and meta-analysis. J Shoulder Elbow Surg 2019;28(04):765-773

37 Seidl A, Sholder D, Warrender W, et al. Early Versus Late Reverse Shoulder Arthroplasty for Proximal Humerus Fractures: Does It Matter? Arch Bone Jt Surg 2017;5(04):213-220

38 Hamilton MA, Diep P, Roche C, et al. Effect of reverse shoulder design philosophy on muscle moment arms. J Orthop Res 2015;33 (04):605-613

39 Verdano MA, Aliani D, Galavotti C, Maroun C, Vaienti E, Ceccarelli F. Grammont versus lateralizing reverse shoulder arthroplasty for proximal humerus fracture: functional and radiographic outcomes. Musculoskelet Surg 2018;102(Suppl 1):57-65

40 Sirveaux F, Roche O, Molé D. Shoulder arthroplasty for acute proximal humerus fracture. Orthop Traumatol Surg Res 2010;96 (06):683-694

41 Barros LH, Figueiredo S, Marques M, Rodrigues C, Ramos J, Claro R. Tuberosity Healing after Reverse Shoulder Arthroplasty for Proximal Humerus Fractures: Is there Clinical Improvement? [Published online: 2020-02-07]. Rev Bras Ortop. Available from: https:// www.thieme-connect.de/products/ejournals/pdf/10.1055/s0039-3402459

42 Schoch B, Aibinder W, Walters J, et al. Outcomes of Uncemented Versus Cemented Reverse Shoulder Arthroplasty for Proximal Humerus Fractures. Orthopedics 2019;42(02):e236-e241

43 Dezfuli B, King JJ, Farmer KW, Struk AM, Wright TW. Outcomes of reverse total shoulder arthroplasty as primary versus revision procedure for proximal humerus fractures. J Shoulder Elbow Surg 2016;25(07):1133-1137

44 Gillespie R, Shishani Y, Joseph S, Streit JJ, Gobezie R. Neer Award 2015: A randomized, prospective evaluation on the effectiveness of tranexamic acid in reducing blood loss after total shoulder arthroplasty. J Shoulder Elbow Surg 2015;24(11):1679-1684

45 Kirsch JM, Bedi A, Horner N, et al. Tranexamic Acid in Shoulder Arthroplasty: A Systematic Review and Meta-Analysis. JBJS Rev 2017;5(09):e3

46 Sun CX, Zhang L, Mi LD, Du GY, Sun XG, He SW. Efficiency and safety of tranexamic acid in reducing blood loss in total shoulder arthroplasty: A systematic review and meta-analysis. Medicine (Baltimore) 2017;96(22):e7015

47 Noguera L, Trigo L, Melero V, Santana F, Torrens C. Reverse shoulder arthroplasty for acute proximal humeral fractures: Postoperative complications at 7 days, 90 days and 1 year. Injury 2019;50(02):371-375

48 Koh J, Galvin JW, Sing DC, Curry EJ, Li X. Thirty-day Complications and Readmission Rates in Elderly Patients After Shoulder Arthroplasty. J Am Acad Orthop Surg Glob Res Rev 2018;2(11):e068

49 Lopiz Y, García-Coiradas J, Serrano-Mateo L, García-Fernández C, Marco F. Reverse shoulder arthroplasty for acute proximal humeral fractures in the geriatric patient: results, health-related quality of life and complication rates. Int Orthop 2016;40(04): 771-781

50 Austin DC, Torchia MT, Cozzolino NH, Jacobowitz LE, Bell JE. Decreased Reoperations and Improved Outcomes With Reverse Total Shoulder Arthroplasty in Comparison to Hemiarthroplasty for Geriatric Proximal Humerus Fractures: A Systematic Review and Meta-Analysis. J Orthop Trauma 2019;33(01):49-57

51 Manoli A, Capriccioso CE, Konda SR, Egol KA. Total shoulder arthroplasty for proximal humerus fracture is associated with increased hospital charges despite a shorter length of stay. Orthop Traumatol Surg Res 2016;102(01):19-24

52 Liu JN, Agarwalla A, Gowd AK, et al. Reverse shoulder arthroplasty for proximal humerus fracture: a more complex episode of care than for cuff tear arthropathy. J Shoulder Elbow Surg 2019;28 (11):2139-2146

53 Osterhoff G, O'Hara NN, D'Cruz J, et al. A Cost-Effectiveness analysis of reverse total shoulder arthroplasty versus hemiarthroplasty for the management of complex proximal humeral fractures in the elderly. Value Health 2017;20(03):404-411

54 Solomon JA, Joseph SM, Shishani Y, et al. Cost Analysis of Hemiarthroplasty Versus Reverse Shoulder Arthroplasty for Fractures. Orthopedics 2016;39(04):230-234 